\title{
OTR Measurements and Modeling of the Electron Beam Optics at the E-cooling Facility
}

\author{
A. Warner ${ }^{1}$, A. Burov ${ }^{1}$, K. Carlson ${ }^{1}$, G. Kazakevich ${ }^{2}$, S. Nagaitsev ${ }^{1}$, \\ L.Prost $^{1}$, M. Sutherland ${ }^{1}$, and M. Tiunov ${ }^{2}$ \\ ${ }^{I}$ Fermi National Accelerator Laboratory, P.O.Box 500, Batavia IL 60543 \\ ${ }^{2}$ Budker Institute of Nuclear Physics, 630090 Novosibirsk, Russia
}

\begin{abstract}
Optics of the electron beam accelerated in the Pelletron, intended for the electron cooling of $8.9 \mathrm{GeV}$ antiprotons in the Fermilab recycler storage ring, has been studied. The beam profile parameters were measured under the accelerating section using Optical Transition Radiation (OTR) monitor. The monitor employs a highly-reflective 2 inch-diameter aluminum OTR-screen with a thickness of $5 \mu \mathrm{m}$ and a digital CCD camera. The measurements were done in a pulse-signal mode in the beam current range of 0.03-0.8 A and at pulse durations ranging from $1 \mu \mathrm{s}$ to $4 \mu \mathrm{s}$. Differential profiles measured in pulsed mode are compared with results obtained by modeling of the DC beam dynamics from the Pelletron cathode to the OTR monitor. The modeling was done with SAM, ULTRASAM and BEAM programs. An adjustment of the magnetic fields in the lenses of the accelerating section was done in the simulations. The simulated electron beam optics downstream of the accelerating section was in good agreement with the measurements made with pulsed beam.
\end{abstract}

Keywords: Optical transition radiation, CCD camera, electron beam, accelerator, bremsstrahlung

PACS: 29.27.Bd, 29.27.Eg, 29.27.Fh

\section{INTRODUCTION}

Optical transition radiation monitors are being used with pulsed beam to image and model the charge distributions of the $4.3 \mathrm{MeV}$ electron beam used in the electron cooler at Fermilab. The transition radiation is produced by the charged particles as they traverse the boundary between media with different dielectric constants [1]. The OTR monitors have several advantages over more traditional imaging devices; they provide an image of the 2-D beam distribution in a plane, they have a linear response to beam charge with good spatial resolution and they have a wide possibility in data processing. Pulsed operation of the electron beam is achieved by modulating the voltage of the gun control electrode and was developed primarily for diagnostics and tuning purposes. To compensate for pulsing and optic variations during the pulse, differential processing of profiles taken at different pulse durations were done. Comparison of the measured beam differential profile dynamics with that of simulated DC beam dynamics show good agreement. 


\section{OTR Beam Profile Monitor Set-up}

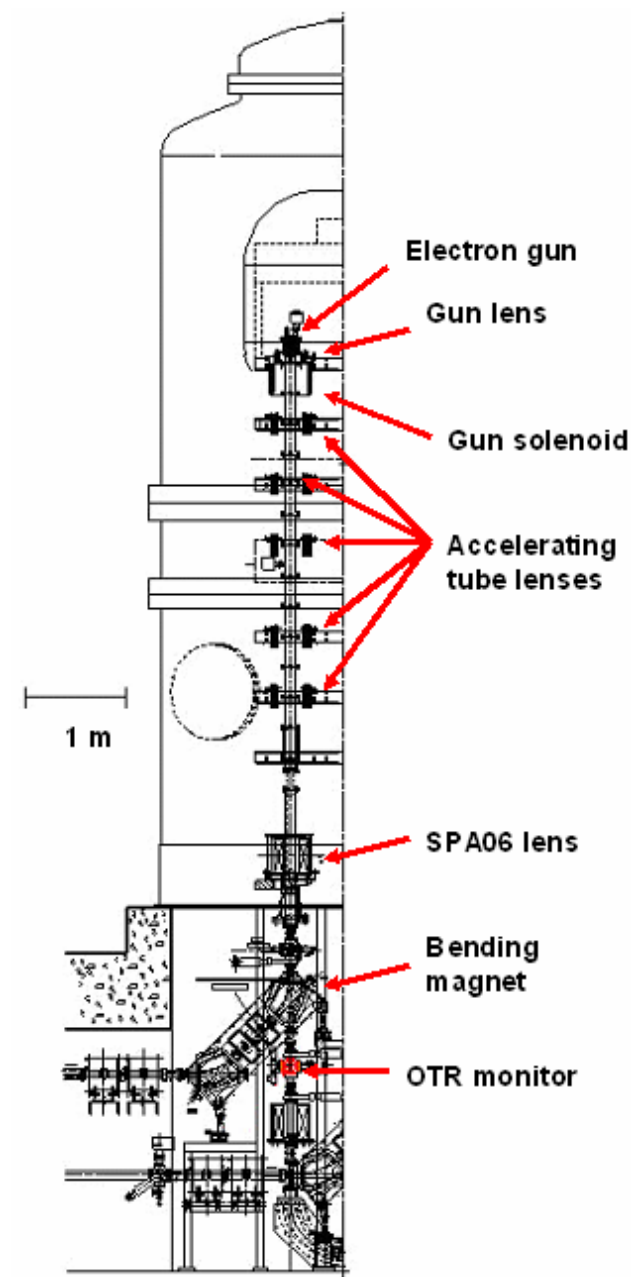

FIGURE 1. Layout of the experimental setup.

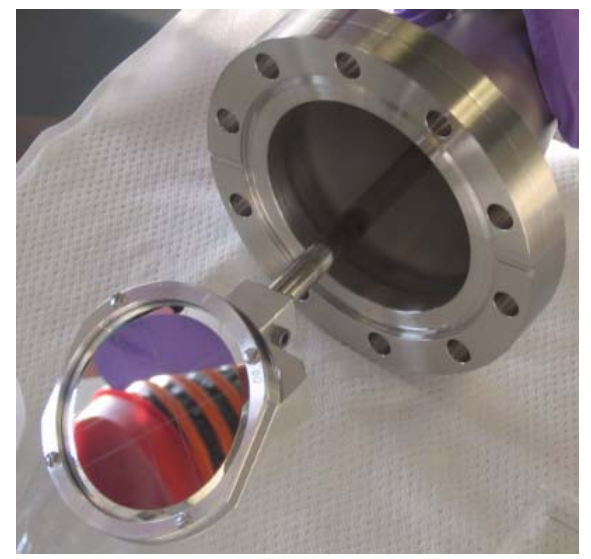

FIGURE 2. OTR screen mount.

The OTR beam profile monitor is mounted in a standard 6-way cross downstream of the accelerating section of the cooling facility, FIG.1, and is composed of a 2"-in diameter OTR screen made from a mirror-surface $5 \mu \mathrm{m}$ aluminum foil tensioned with high flatness onto a ring frame movable with a stepper-motor actuator. Such a thin foil is essential to reduce the background caused by beam scattering and bremsstrahlung. The linear drive provides insertion of the OTR screen with accuracy of $0.1 \mathrm{~mm}$ and the screen is inserted into the beam at an angle of 38.9 degrees to the beam direction. This corresponds to the angle $\theta \approx 1 / \beta \gamma$ [2], where the intensity of the light has a maximum. This allows to significantly increase the monitor sensitivity and to operate with low beam current. A photo of the OTR screen mounted on the actuator is shown in FIG. 2.

The backward OTR passes through the vacuum glass window to the digital CCD camera that provides an image the beam. To transform images to real-world coordinates, four $75 \mu \mathrm{m}$ tungsten wires are mounted over the radiation screens to form a rectangular $10 \mathrm{~mm} \times 10 \mathrm{~mm}$ grid. The CCD camera has an objective with: focal length $\mathrm{f}=25 \mathrm{~mm}$; relative aperture $F=1.4$. The distance between the OTR screen and the camera lens is of $320 \mathrm{~mm}$. The CCD camera and associated optics are incased in a light tight housing to omit external sources of light.

The digital camera is connected to computer via a IEEE 1394 fire-wire interface. A Labview based data acquisition and processing systems have been developed to obtain with real-time the beam spot images and to store them. The processing system provides the operator with tools for image analysis. The OTR diagnostic system employed at the cooler is designed to be 
routinely used to optimize the beam transport and to measure the transverse beam profiles and the respective beam sizes.

\section{Data Acquisition}

The images taken from the optical monitors are digitized in the CCD camera and saved in an uncompressed format. As a result external electronic and environmental noise sources have little effect on the image quality. In addition, the IEEE 1394 firewire interface allows control of the camera's gain and other features remotely. The images are then displayed and analyzed with application software that was developed using LabView and IMAQ vision utility tools. The program allows the user to display live (real-time) images of the beam and to simultaneously analyze either current (live) data or stored data from files. Two profiles taken with different pulse durations or optical settings can therefore be compared. This type of development differs from the standard application in that it incorporates image digitization, image display, as well as image analysis and system calibration in a real-time module. These image analysis tools are used to combine techniques that compute statistics and measurements based on the gray-level intensities of the image pixels; linear (convolution) filters can be applied to remove unwanted background at any stage of the analysis if necessary.

\section{Results of the Measurements}

The system so far described has been used to measure the properties of the electron beam while the Pelletron was operating in a pulse mode with the bending magnet turned off. This allows accelerated beam to be passed straight onto the OTR monitor. The residual field in the bending magnet was compensated with a coil mounted in the bending magnet. Images of $1 \mu$ s beam pulses were subtracted from $2 \mu$ seam pulses to compensate for pulser and gun optics variations during the pulse. The electron beam was aligned with BPM monitors located upstream of lens SPA06 and downstream of the OTR monitor before all measurements to avoid passing the beam off axis through the OTR.

During the measurements we checked the response of the monitor versus beam current which was varied by changing the pulse voltage of the gun control electrode. FIG. 3 shows the dependence of the integral of detected light in the beam spot versus the beam current and demonstrates good linearity of the OTR monitor. Moreover we measured dependence of the monitor sensitivity versus position of the beam along both the $\mathrm{X}$ and $\mathrm{Y}$ axes of the screen at a fixed beam current. Motion of the beam along the axes was done using correctors inside the focusing lens SPA06, FIG. 1.

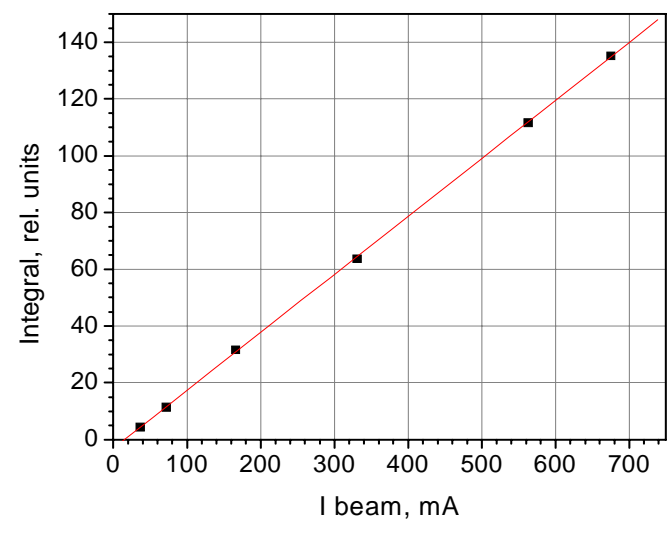

FIGURE 3. Beam spot integrals versus the beam current. 
The measured dependence of the sensitivity on the beam position over the surface area of the screen is relatively weak; as a result this allows the use of the full area of the screen for the beam monitoring.

The beam profiles were measured as functions of the current of the lens SPA06 with a beam current of 0.56 A. FIG. 4 shows the variation of the profiles versus the lens current. One can see that the beam profiles are dissimilar with various lens current.
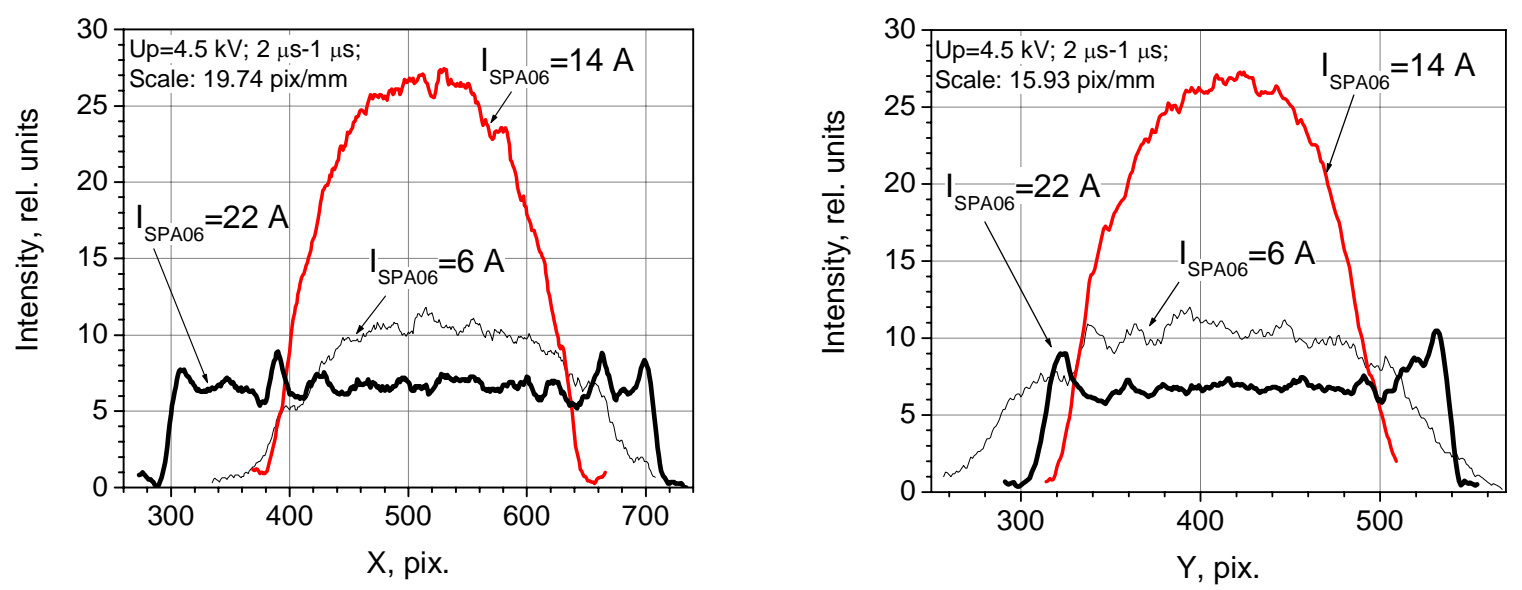

FIGURE 4. Beam profiles versus current in lens SPA06.

\section{Simulation Results}

The beam profiles plotted in FIG. 4 can not be explained as a trivial effect of focusing with SPA06 lens. We therefore studied this phenomenon with simulations of the beam optics in DC mode from the cathode to the OTR monitor with space charge effects included. The accuracy of the simulations was improved by using fitted magnetic fields in the lenses of the accelerating section. The fitting of the fields were done with SAM code package [3] and the measured distributions of the fields.

Simulation of the beam ${ }_{R, m m}$ dynamics and optics in the electron gun was done with the 2D ULTRASAM code [4]. The results of the simulation, including the trajectories of the electrons, the contours of electrodes, the equi-potentials, and the distribution of the electric and magnetic fields are

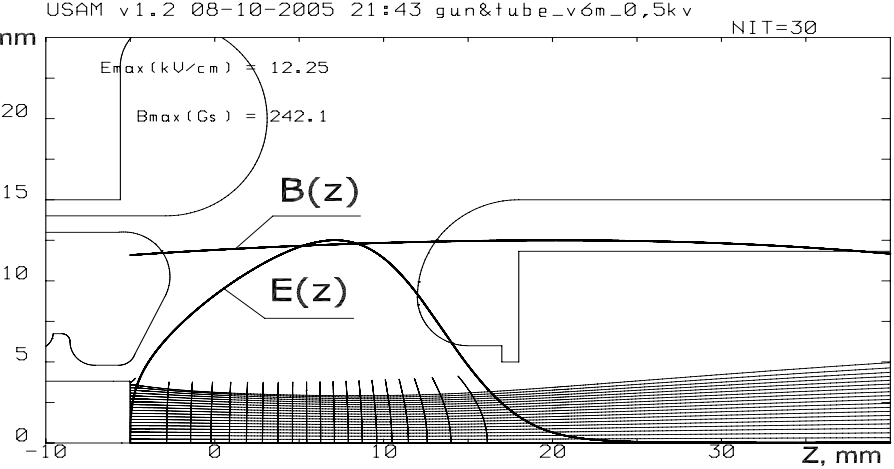

FIGURE 5. Results of the electron gun optics calculation. shown in FIG. 5.

The beam optics from the exit of the gun to the OTR-monitor was calculated with the BEAM program [5] using the gun exit beam parameters from the ULTRASAM code as input. The calculated electron beam envelopes versus SPA06 current for full 
beam current and 25\% of the beam current, respectively, are shown in FIG. 6 (left) together with the corresponding distribution of the axial magnetic fields. The figure demonstrates that the similarity in the beam profiles along the beam axis is not conserved. Such phenomenon can be explained as a nonlinear dependence of the transverse velocities in the beam versus the radial coordinate for the existing gun optics; an inhomogeneous current density distribution in the gun leads to similar results. Note that with a linear profile of transverse velocities and a homogeneous current density the ratio of the radii for the $25 \%$ and $100 \%$ beam envelopes is a constant along the $\mathrm{Z}$ axis.

Fig.6 (right) shows measured (dots) and calculated (solid lines) beam X-profiles for the SPA06 lens current values of 6A, 14A, and 22A. The measured Y-profiles also approximately coincide with calculated profiles considering the slightly elliptic shape of the real beam. All measured and calculated profiles demonstrate good agreement.
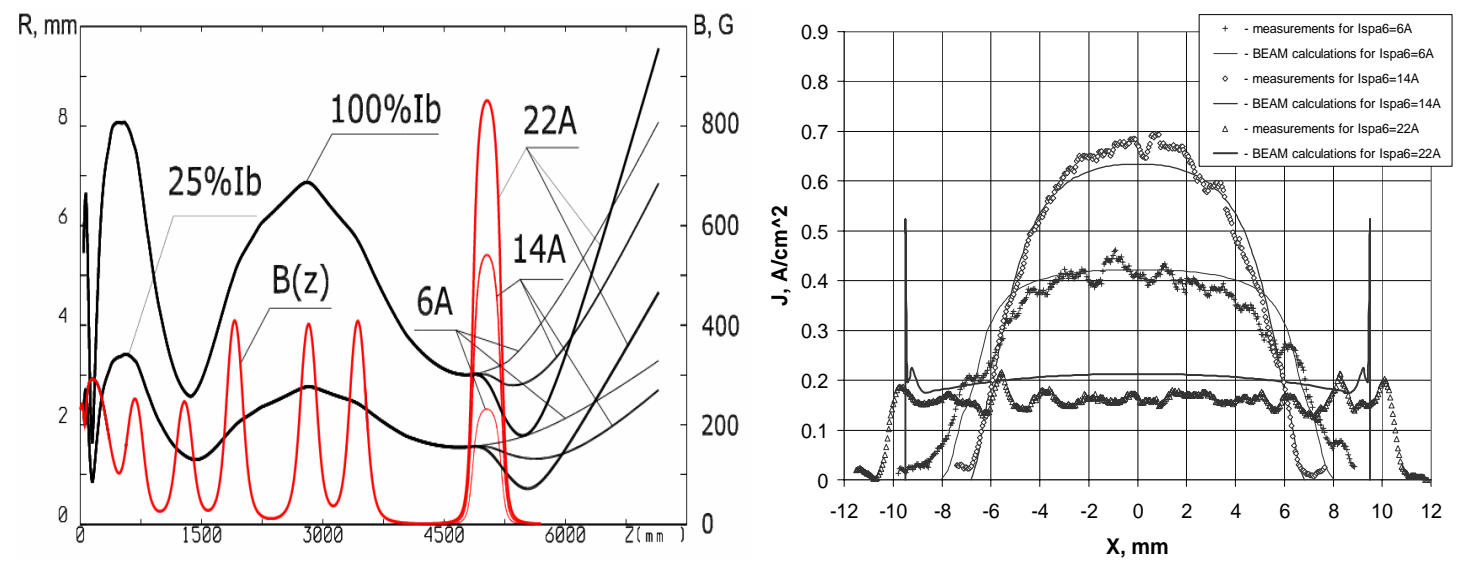

FIGURE 6. Beam envelopes and beam profiles versus SPA06 current.

\section{SUMMARY}

OTR monitoring, data acquisition and processing have been developed for analysis of beam optics with the Pelletron in the pulse mode. Using those systems measurements were done of the beam profiles downstream of the Pelletron accelerating section at the OTR screen location. Modeling of the beam optics in DC mode from the cathode to the OTR screen was done with SAM, ULTRASAM and BEAM programs. The measured result show good agreement with the modeling and also explains the variation of the beam profiles versus the SPA06 lens current.

\section{ACKNOWLEDGMENTS}

The authors would like to express appreciations to the team of expert technicians that worked with us to assemble and install all the systems, especially: R.Kellet, A.Germain, J.Nelson, W.Johnson, F.Juarez and M.Frett. 


\section{REFERENCES}

1. V.L. Ginzburg and I.M. Frank, "Radiation from a Uniformly Moving Electron passing from One Medium to Another”, Journ. of Experimental and Theoretical Physics (JETP) V.16, pp. 15-26 (1946).

2. A. Warner, G. Kazakevich,S. Nagaitsev, G. Tassotto, W. Gai and R. Konecny "Beam Profile Diagnostics for the Fermilab Medium Energy Electron Cooler.” IEEE Trans.Nucl.Sci, V. 52, No 5, 2005

3. B.M. Fomel, M.A. Tiunov, V.P. Yakovlev. SAM - an Interactive Code for Evaluation of Electron Guns. Preprint BINP 96-11, Novosibirsk, 1996.

4. A.V. Ivanov, M.A. Tiunov. ULTRASAM - 2D Code for Simulation of Electron Guns with Ultra High Precision. Proceeding of EPAC-2002, Paris, 2002.

5. M.A. Tiunov. BEAM - 2D-code package for simulation of high perveance beam dynamics in long systems. Proceedings of International Symposium "SPACE CHARGE EFFECTS IN FORMATION OF INTENSE LOW ENERGY BEAMS". February 15-17, 1999, JINR, Dubna, Russia 